\title{
The expression of BMP4 during the hematopoiesis of human yolk sac
}

\author{
Yuesi Wang ${ }^{1}$, Hui $\mathrm{Xu}^{1}$, Jianyuan $\mathrm{Li}^{2}$, Haiyan $\mathrm{Wan}^{2}$, Shaohua $\mathrm{Jin}^{2}$ \\ ${ }^{1}$ Department of Biotech, Binzhou Medical College, Yantai 264003, China; ${ }^{2}$ Shandong Research Center of S tem Cell Engineering, \\ Yantai 264000, China
}

In the mammal, primitive hematopoiesis is first derived from mesodermal cells within a region out of the embryonic sculture known as the yolk sac. Within this region, primitive red cells and hematopoietic stem cells (HSCs) are thought to arise from precursors located in the ventral wall of the yolk sac. However, the factors that regulate HSC development in vivo are still largely unknown. Some Study showed that exogenously added Bone morphogenetic proteins 4 regulate HSC proliferation, differentiation, and survival. To further explore the mechanism by which BMP4 might be involved in hemopoietic differentiation of yolk sac, we observed the expression of BMP4,CD34, CD133 and tyrosine kinase receptors (KDR) in the blood island of yolk sac at embryonic 3 to 12 weeks, Gene expression was analyzed by RT-PCR and the presence of BMP4, CD34, CD133 and KDR protein were confirmed by immunohistochemistry (IHC). In the human yolk sac, we find that BMP-4 is expressed at high levels from the 16th day to the 7 week, and decreased quickly after week 7. The results showed that KDR, CD133 and CD34 largely appeared on the 21 stand 30th day, then increased at the 6th weeks, and decreased quickly after week 7 too. Furthermore, Ihh, SCI, GATA-1, GATA-2 and PU.1 mRNAs were showed that PU.1 was not expressed on the 16th days, other factors were expressed all the time. Thus, the distribution of BMP4, KDR, CD34, CD133 and transcription factors expression are highly suggested that BMP4 was secreted from yolk sac which might exert its effects on the specification of human hemangioblast and hematopoietic stem cells from embryonic mesoderm in vivo through transcription factors.

Keywords: yolk sac, hemangioblasat, hematopoietic stem cell, BMP-4, human embryo

Cell Research (2008) 18:s50. doi: 10.1038/cr.2008.140; published online 4 August 2008

Correspondence:Yuesi Wang

E-mail: wys74161@163.com 